\title{
Effect of Seizure During Pregnancy on Pentylenetetrazol-Induced Seizure-Like Behavior and Serum GDNF Levels in Adult Male Mice Offspring
}

\author{
Ayoob Sabaghi ${ }^{1}$, Ali Heyrani ${ }^{*}$, Amir Kiani ${ }^{2}$, Namdar Yousofvand ${ }^{3}$ \\ ${ }^{1}$ Faculty of Physical Education and Sport Sciences, Razi University, Kermanshah, Iran \\ ${ }^{2}$ Faculty of Pharmacology, Kermanshah University of Medical Sciences, Kermanshah, Iran \\ ${ }^{3}$ Faculty of Sciences, Razi University, Kermanshah, Iran
}

Article Info:

\section{ABSTRACT}

Introduction: Epilepsy is a neurodevelopmental disorder and prenatal factors exert a profound influence on the development of the nervous system in the offspring. Therefore, this study was designed to investigate the effect of seizure during pregnancy on pentylenetetrazol (PTZ)-induced seizure-like behavior in adult male mice offspring. Materials and Methods: After pregnancy, mice were classified as: i) naive group; ii) seizure group, induction of seizure between the fourteenth and nineteenth days of pregnancy by application of PTZ; iii) sham group, received normal saline intraperitoneally during the fourteenth and nineteenth days of pregnancy; and iv) control group, pregnant mice without injection during pregnancy. At postnatal day 95, serum glial cell-derived neurotrophic factor (GDNF) levels and seizure susceptibility to PTZ in male offspring of all groups were evaluated. Results: The results showed that serum GDNF levels and seizure severity of offspring of mice in the seizure group were significantly higher than male offspring in the other group. Conclusion: The present findings showed that seizure during pregnancy may enhance seizure susceptibility in adult male offspring, possibly via increasing serum GDNF.

\section{Key words:}

1. Seizures

2. Pregnancy

3. Mice

4. Glial Cell Line-Derived

Neurotrophic Factor

*Corresponding Author: Ali Heyrani

E-mail: iliaheirani2004@gmail.com 


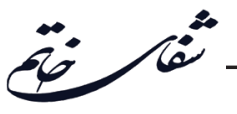

تأثير تشنج در دوران باردارى بر رفتار تشنجى القاشده با پنتيلن تتراوزل و سطوح سرمى GDNF در

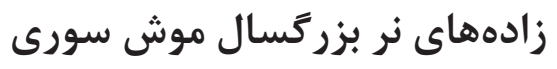

\author{
ايوب صباغى'، على حير انى"، امير كيانى '، نامدار يوسفوند" \\ 'دانشكده تربيت بدنى و علوم ورزشى، دانشعاه رازى، كرمانشاه، ايران

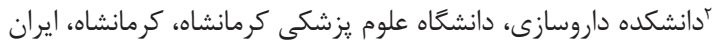

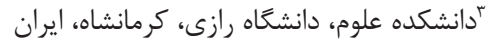

\title{
اطلاعات مقاله:
}

\section{جִ}

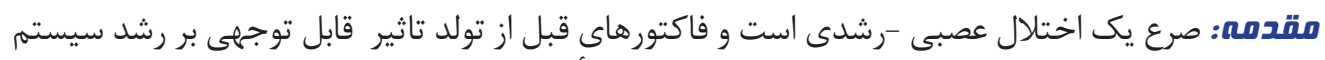

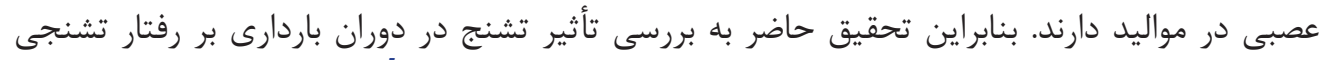

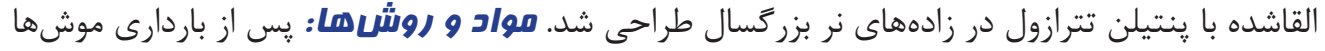

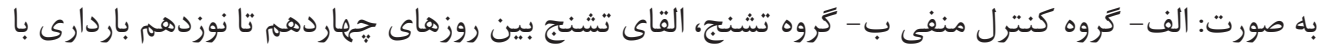

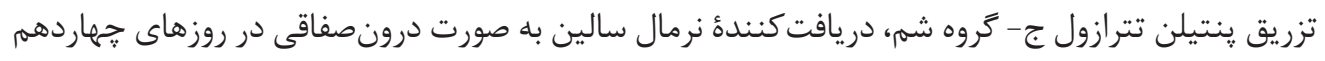

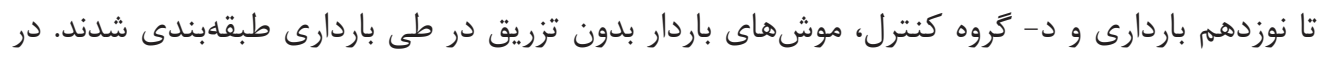

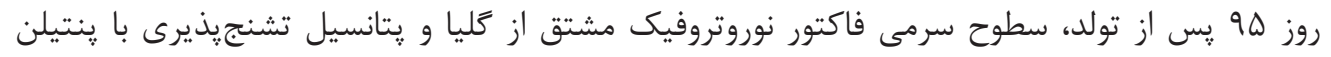

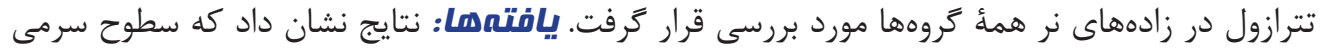

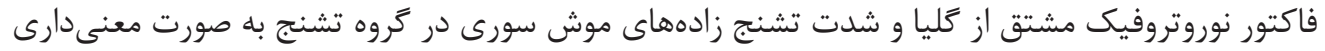

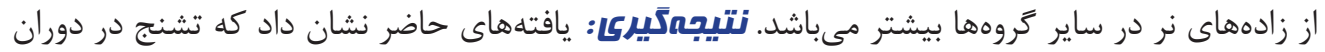

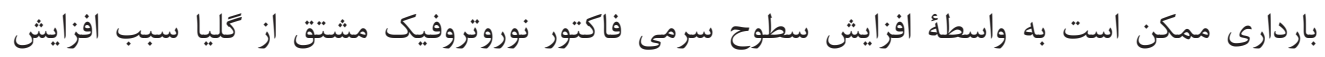
يتانسيل تشنجيذيرى در زادههاى نر بزر خسال شود.

كليد وازمها: 1. r r. rاردارى 


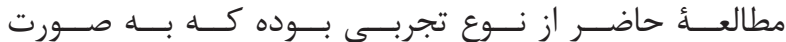

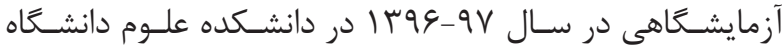

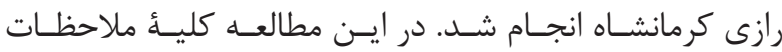

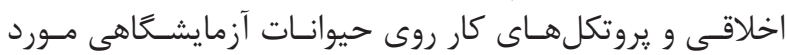

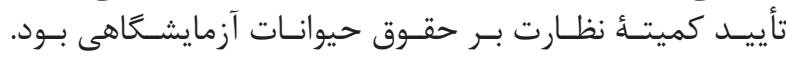

$$
\text { حيوانات مورد آزمايش }
$$

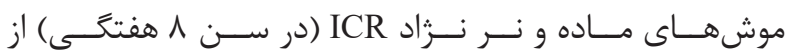

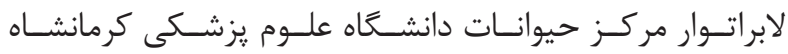

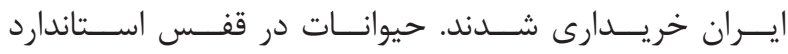

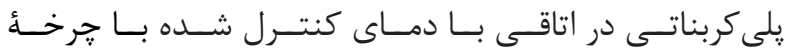

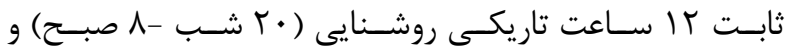

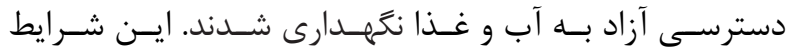

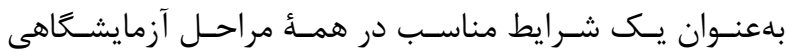

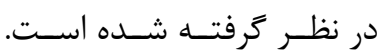

$$
\text { فرايند كيندل كردن }
$$

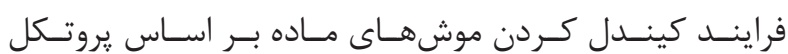

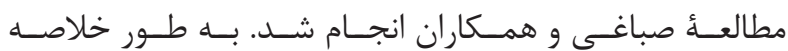

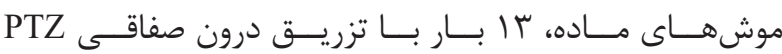

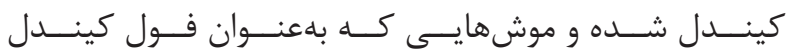

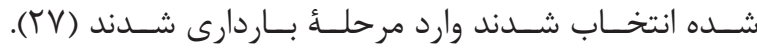

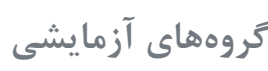

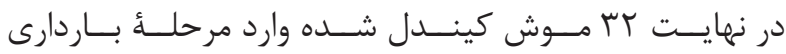

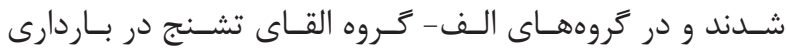

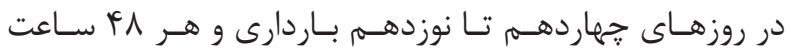

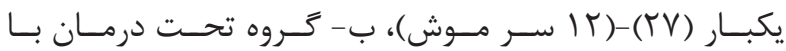

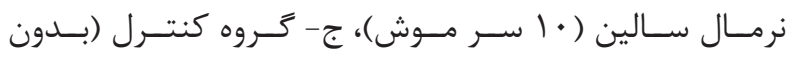

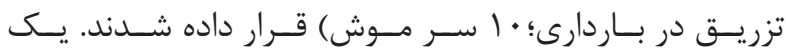

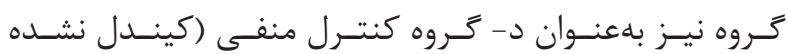

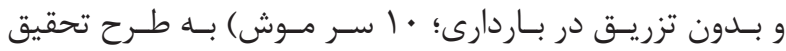

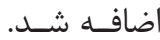

اندازهَيرى سطوح سرمى GDNF

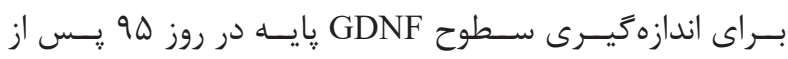

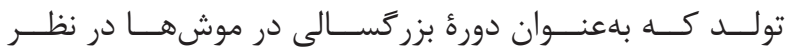

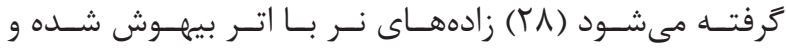

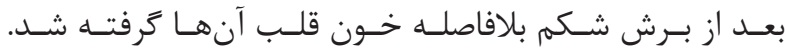

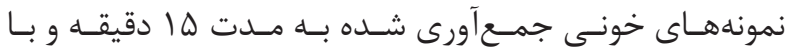

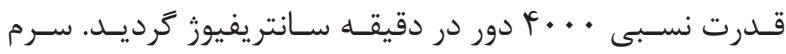

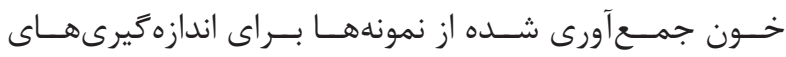

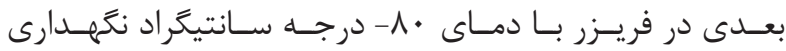

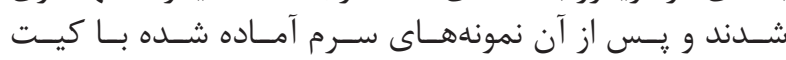

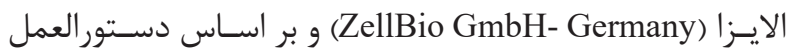

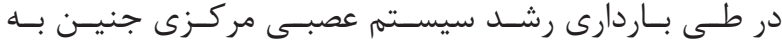

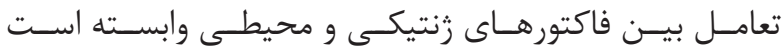

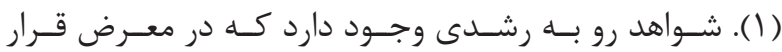

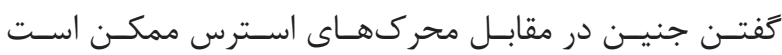

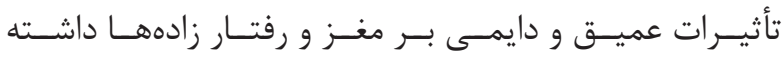

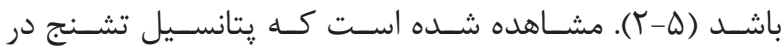

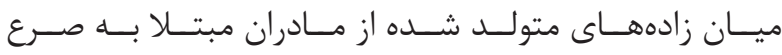

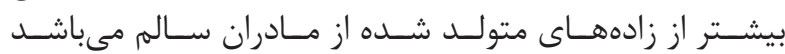

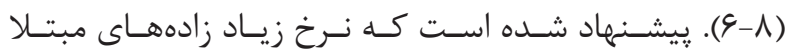

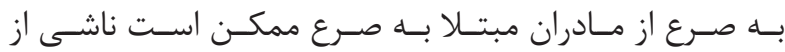

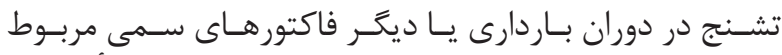

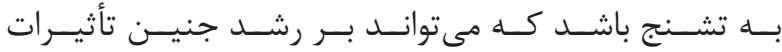

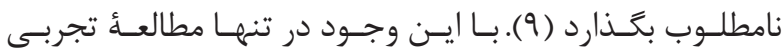

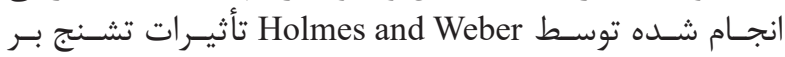

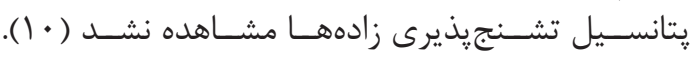

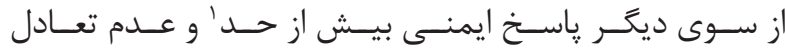

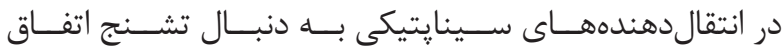

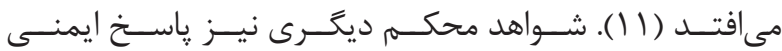

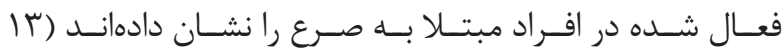

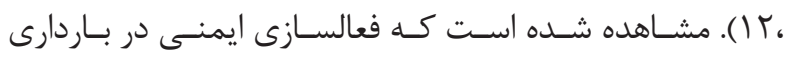

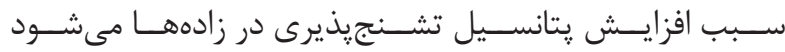

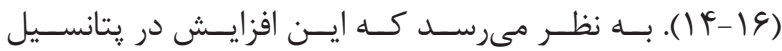

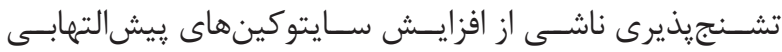

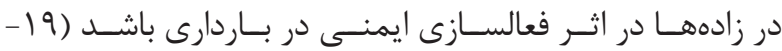

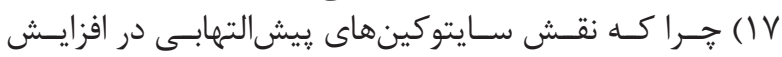

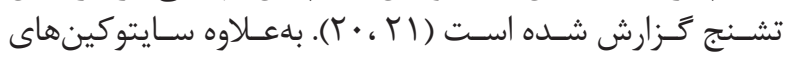

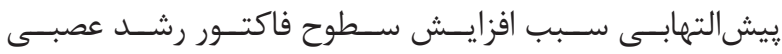

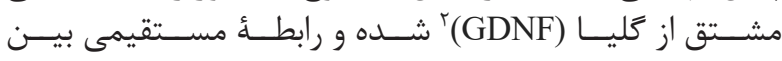

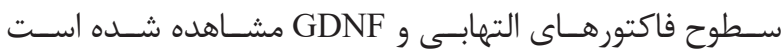

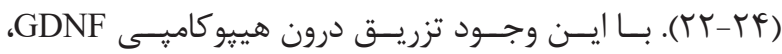

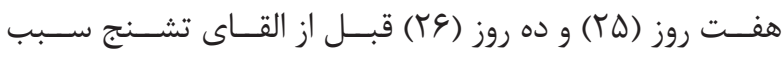

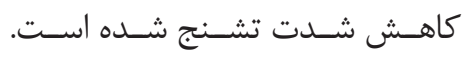

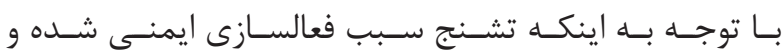

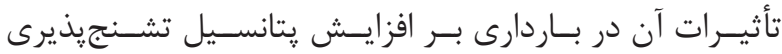

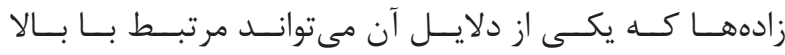

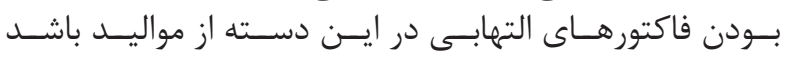

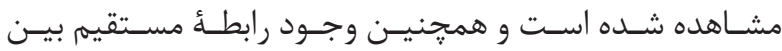

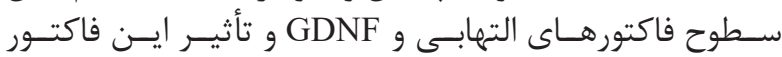

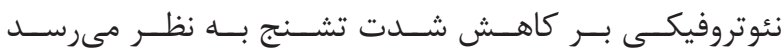

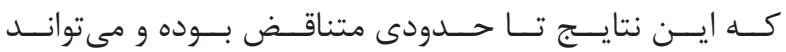

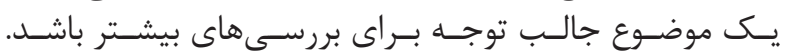

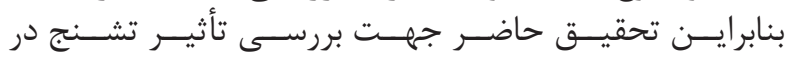

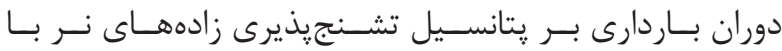

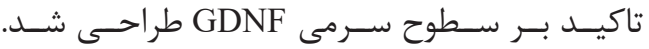




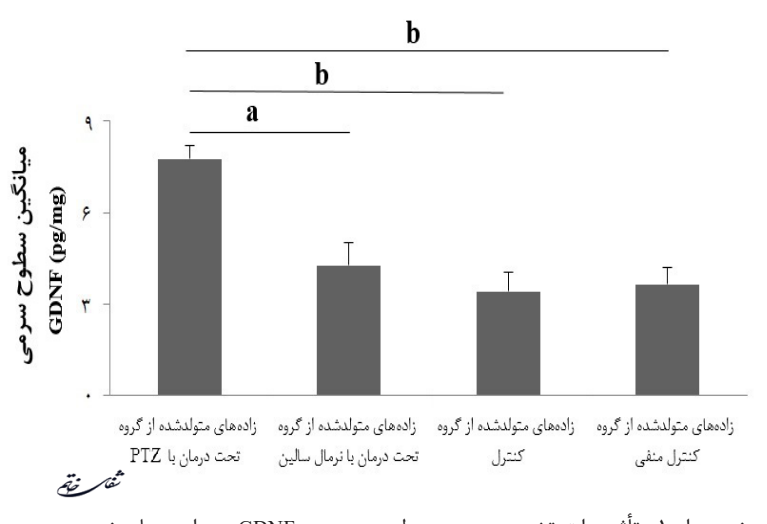

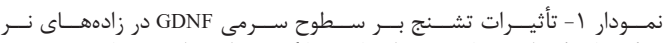

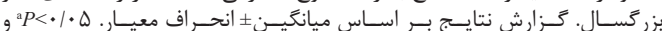

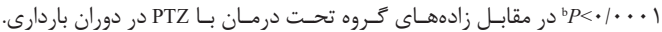

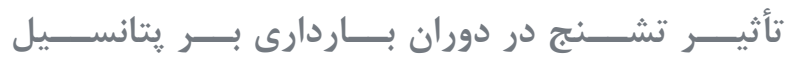

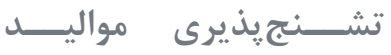

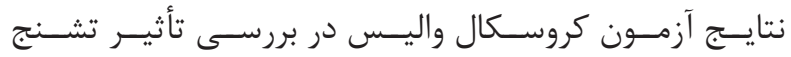

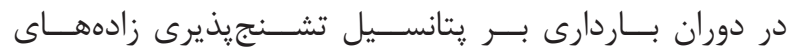

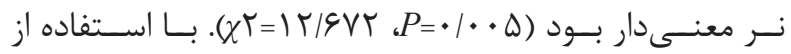

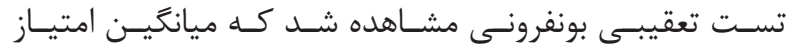

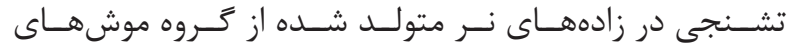

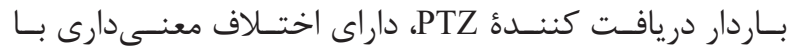

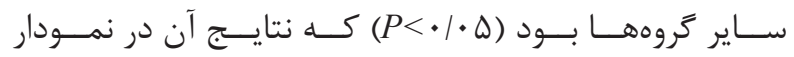

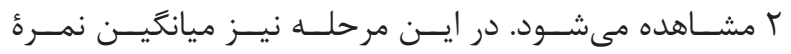

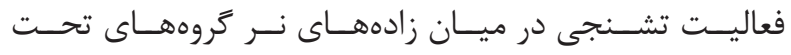

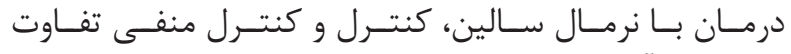

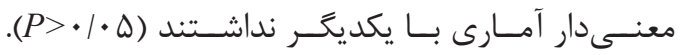

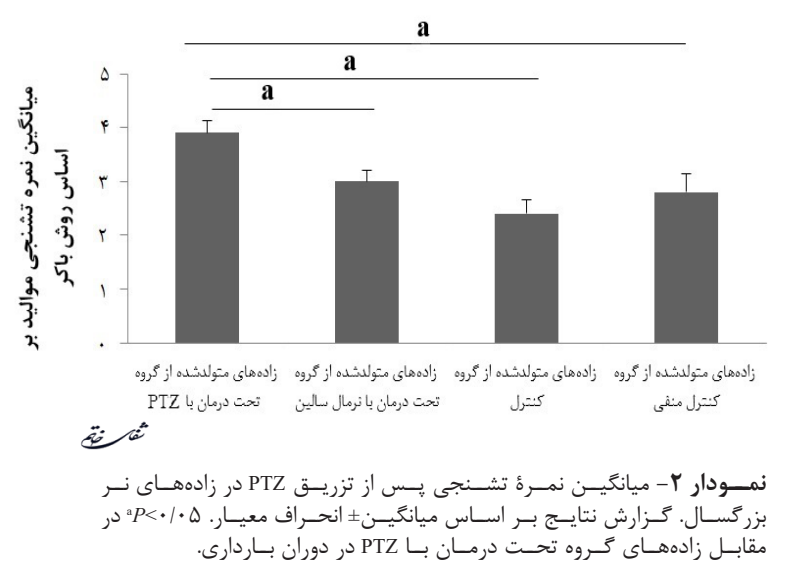

بحث و نتيجهَيرى

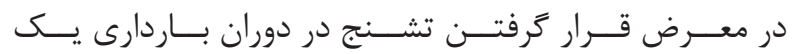

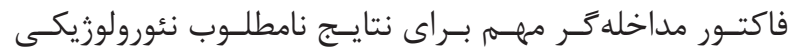

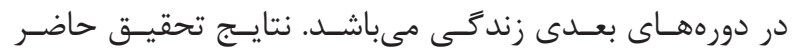

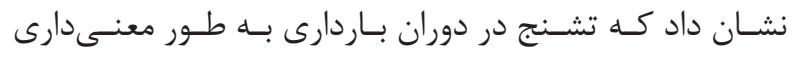

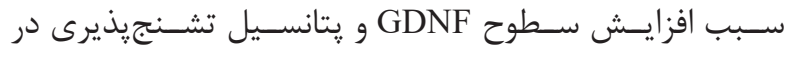

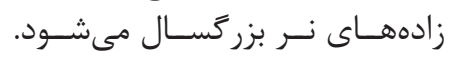

${ }^{3}$ No response

${ }_{5}^{4}$ Ear and facial twitching

${ }^{5}$ Myoclonic jerks without rearing

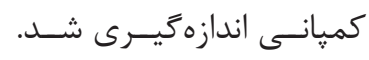

آزمون پِتانسيل تشنج يذيرى

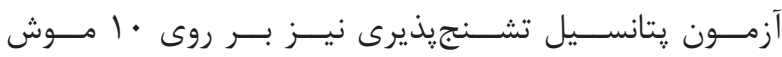

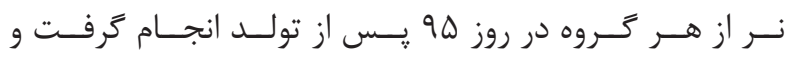

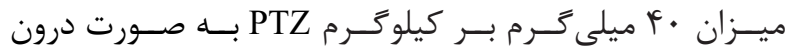

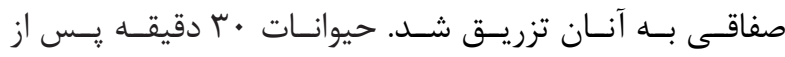

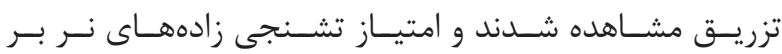

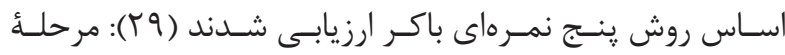

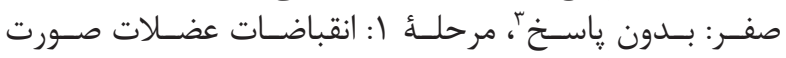

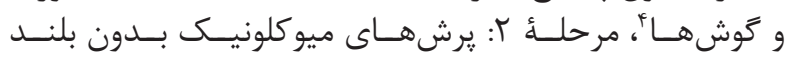

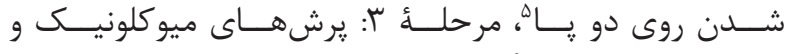

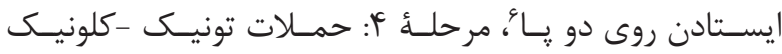

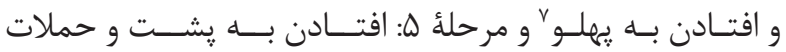

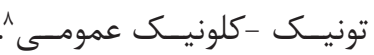

آناليز آمارى توني

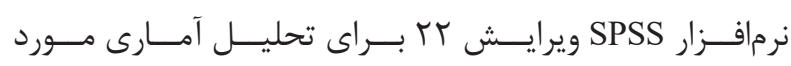

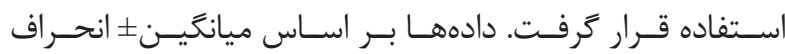

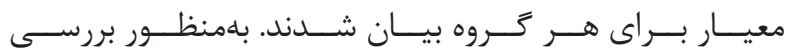

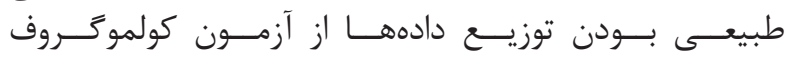

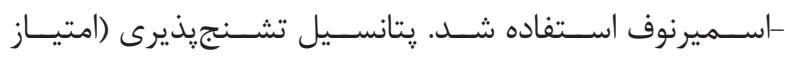

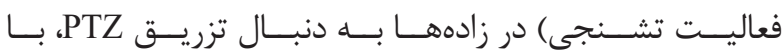

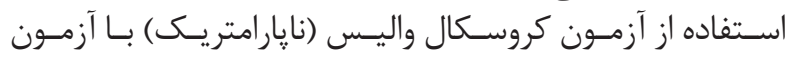

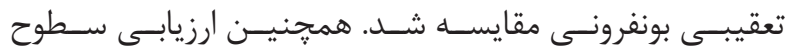

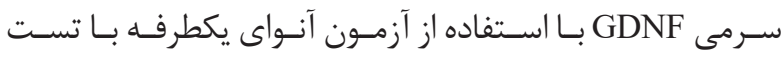

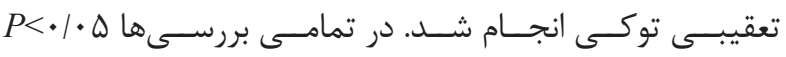

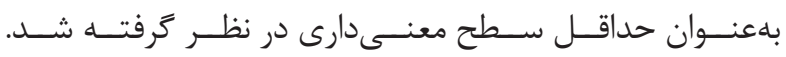

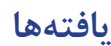

تأثيـــر تشــنج در دوران بــاردارى بــر ســوح ســـــى

GDNF

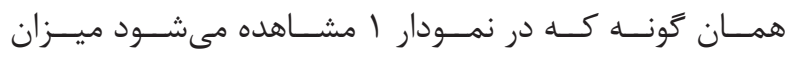

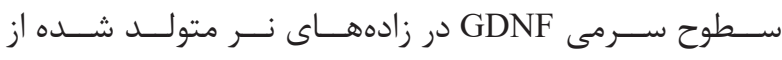

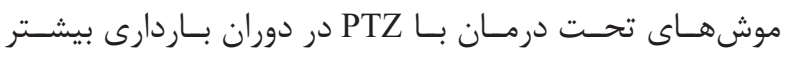

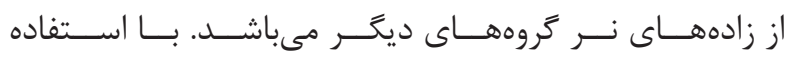

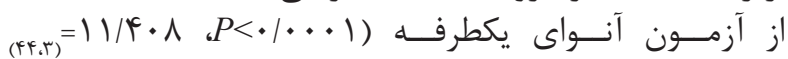

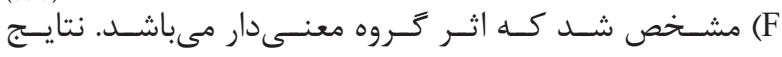

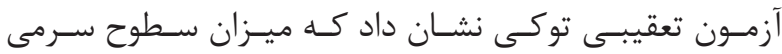

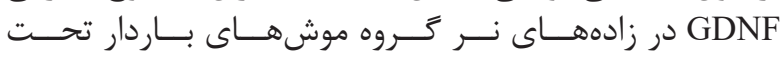

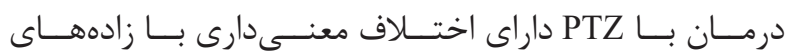

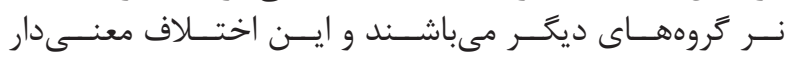

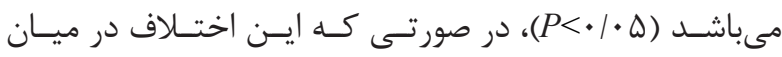

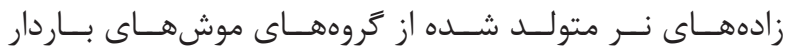

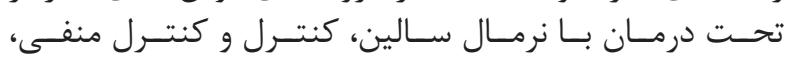

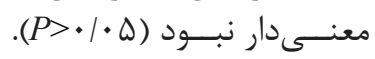

${ }^{6}$ Myoclonic jerks, rearing

${ }^{7}$ Turn over into side position, clonic-tonic seizures

${ }^{8}$ Turn over into back position, generalized clonic-tonic seizures 


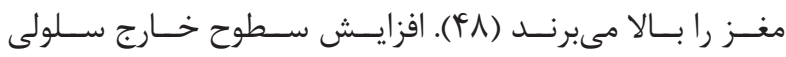

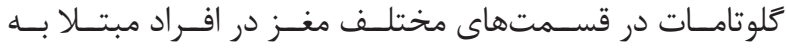

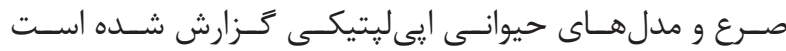

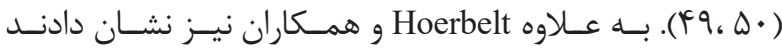

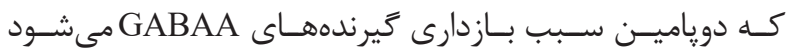

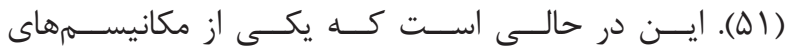

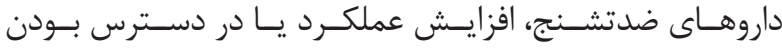

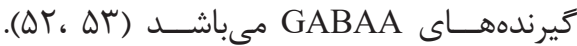

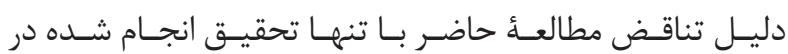

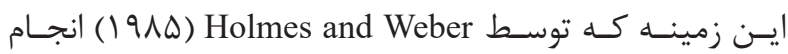

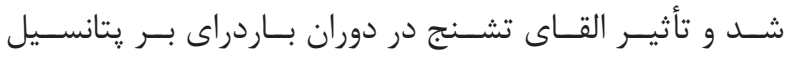

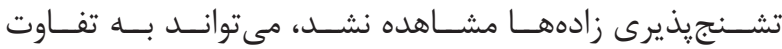

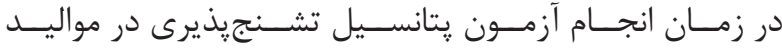

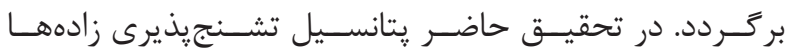

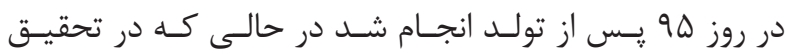
(191ه) Holmes and Weber

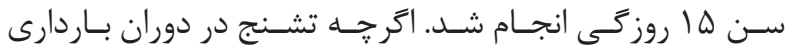

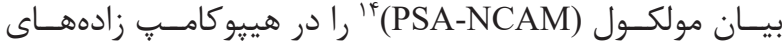

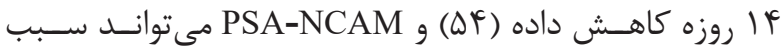

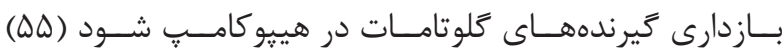

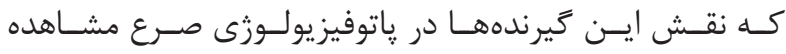

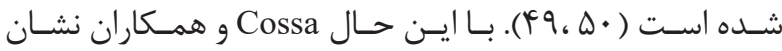

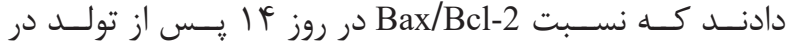

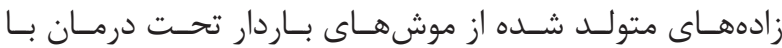

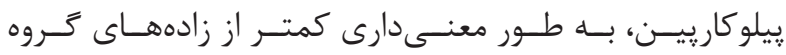

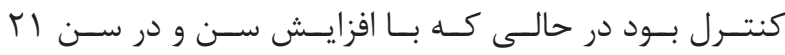

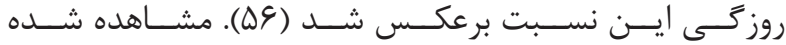

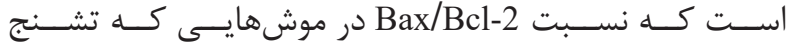

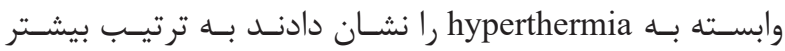

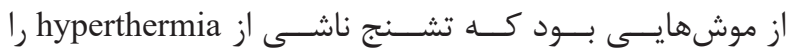

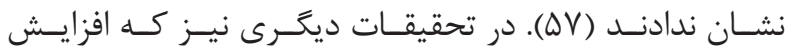

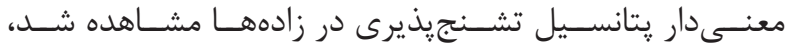

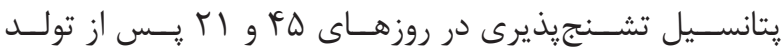

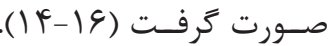

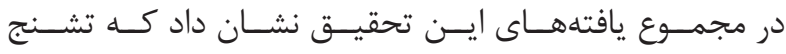

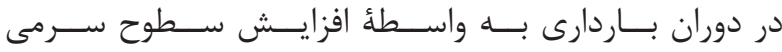

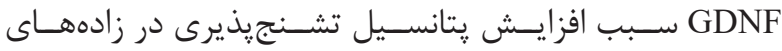

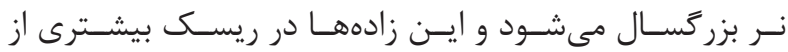

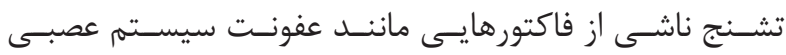

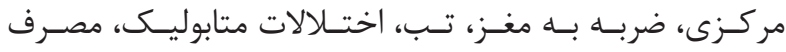

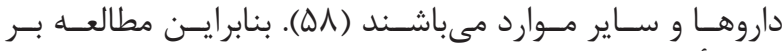

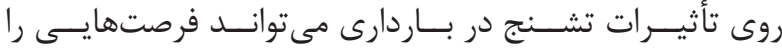

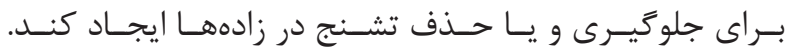

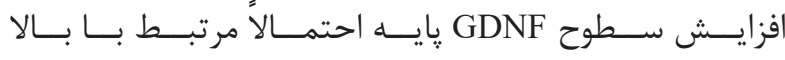

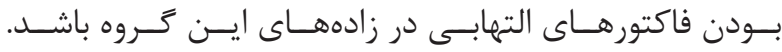

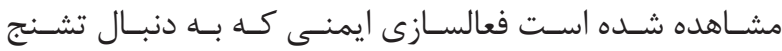

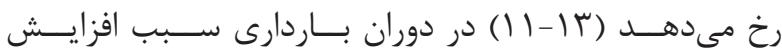

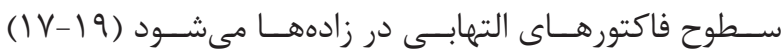

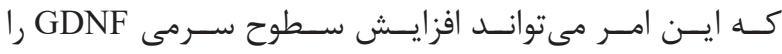

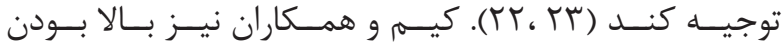

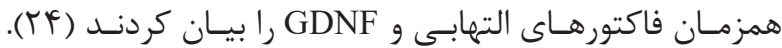

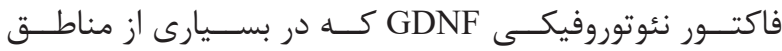

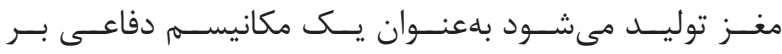

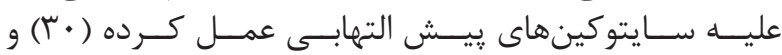

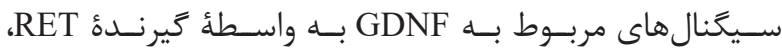

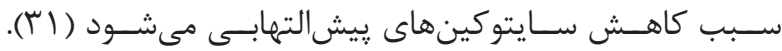

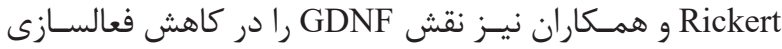

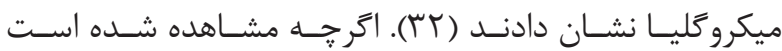

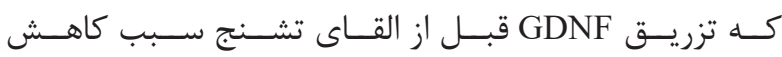

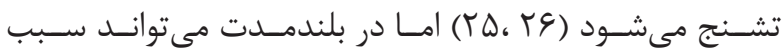

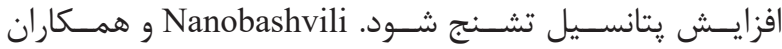

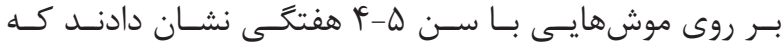

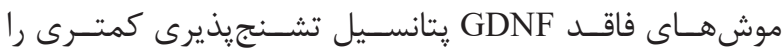

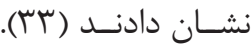

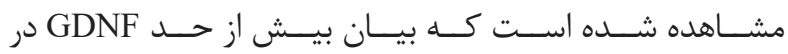

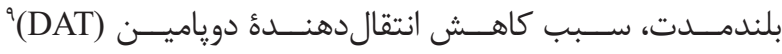
مى

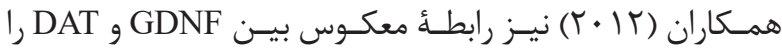

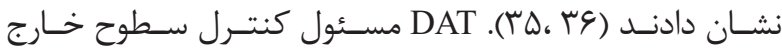

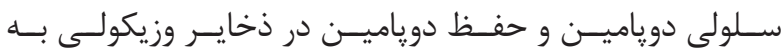

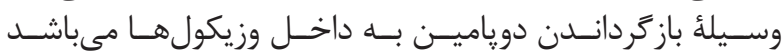

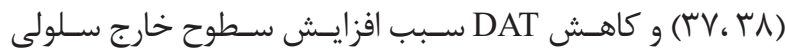

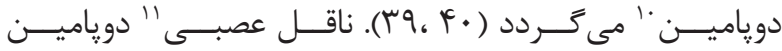

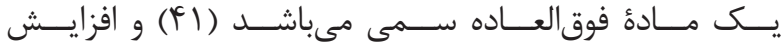

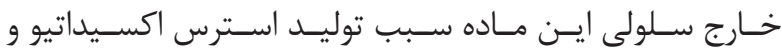

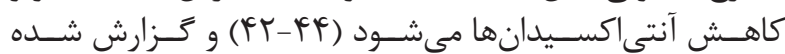

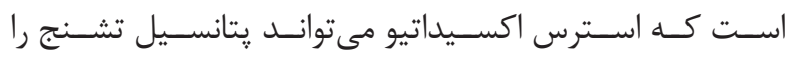

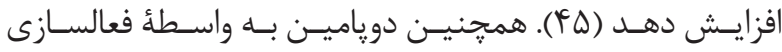

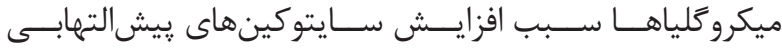

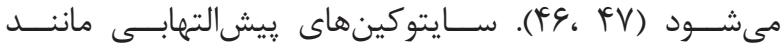

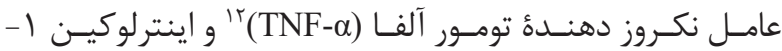

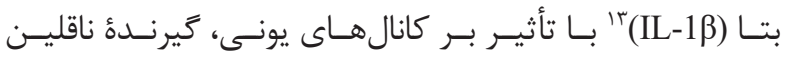

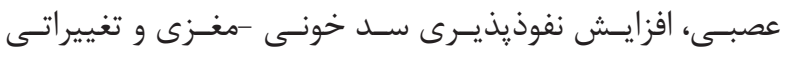

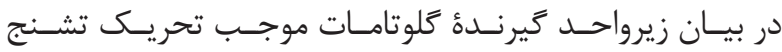

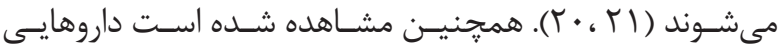

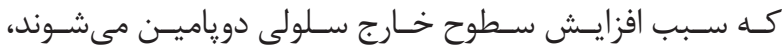

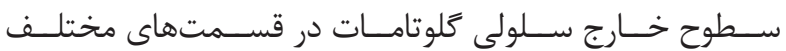

${ }^{9}$ Dopamine transporter

${ }^{10}$ Extracellular dopamine levels

${ }^{11}$ Neurotransmitter
${ }^{12}$ Tumor necrosis factor alpha

${ }^{13}$ Interleukin- $1 \beta$

${ }^{14}$ Polysialylated neural cell adhesion 
1. Heinrichs SC. Neurobehavioral consequences of stressor exposure in rodent models of epilepsy. Prog Neuropsychopharmacol Biol Psychiatry. 2010; 34(5): 808-15.

2. Hagberg H, Gressens P, Mallard C. Inflammation during fetal and neonatal life: implications for neurologicand neuropsychiatric disease in children and adults. Ann Neurol. 2012; 71(4): 444-57.

3. Malkova NV, Yu CZ, Hsiao EY, Moore MJ, Patterson PH. Maternal immune activation yields offspring displaying mouse versions of the three core symptoms of autism. Brain Behav Immun. 2012; 26(4): 607-16.

4. Oskvig DB, Elkahloun AG, Johnson KR, Phillips TM, Herkenham M. Maternal immune activation by LPS selectively alters specific gene expression profiles of interneuron migration and oxidative stress in the fetus without triggering a fetal immune response. Brain Behav Immun. 2012; 26(4): 623-34.

5. Rico JL, Ferraz DB, Ramalho-Pinto FJ, Morato S. Neonatal exposure to LPS leads to heightened exploratorynactivity in adolescent rats. Behav Brain Res. 2010; 215(1): 102-9.

6. Peljto AL, Cummings Ch B, Vasoli VM, Leibson CL, Hauser WA, Buchhalter JR, et al. Familial risk of epilepsy: a population-based study. Brain. 2014; 137(3): 795-805.

7. Babtain FA. Impact of a family history of epilepsy on the diagnosis of epilepsy in Southern Saudi Arabia. Seizure. 2013; 22(7): 542-7.

8. Najafi MR, Najafi MA, SafaeiA. Association of family history of epilepsy with earlier age onset of juvenile myoclonic epilepsy. Iranian Journal of Child Neurology. 2016; 10(2): 10-15.

9. Andersen VE. Genetics in the epilepsies. Trends Neurosci. 1985; 8: 513-6.

10. Holmes GL, Weber DA. Effect of seizures during pregnancy on seizure susceptibility in offspring. Epilepsia. 1985; 26(5): 421-3.

11. Kan AA, de Jager W, de Wit M, Heijnen C, van Zuiden M, Ferrier C, et al. Protein expression profiling of inflammatory mediators in human temporal lobe epilepsy reveals co-activation of multiple chemokines and cytokines. J Neuroinflammation. 2012; 9: 207. doi: 10.1186/1742-2094-9-207.

12. Aronica E, Gorter J. Gene expression profile in temporal lobe epilepsy. Neuroscientist. 2007; 13(2): 100-8.

13. Ravizza T, Gagliardi B, Noe F, Boer K, Aronica E, Vezzani A. Innate and adaptive immunity during epileptogenesis and spontaneous seizures: evidence from experimental models and human temporal lobe epilepsy. Neurobiol Dis. 2008; 29(1): 142-60.

14. Yin P, Liu J, Li Z, Wang YY, Qiao NN, Huang $\mathrm{SY}$, et al. Prenatal immune challenge in rats increases susceptibility to seizure-induced brain injury in adulthood. Brain Res. 2013; 26(1519): 78-86.

15. Yin P, Zhang XT, Li J, Yu L, Wang JW, Lei GF, et al. Maternal immune activation increases seizure susceptibility in juvenile rat offspring. Epilepsy Behav. 2015; 47: 93-7.

16. Magni DV, Souza MA, Oliveira AP, Furian AF, Oliveira MS, Ferreira J, et al. Lipopolysaccharide enhances glutaric acid-induced seizure susceptibility in rat pups: behavioral and electroencephalographic approach. Epilepsy Res. 2011; 93(2-3): 138-48.

17.ZagerA, PeronJP, MennecierG, Rodrigues SC, Aloia TP, Palermo-Neto J. Maternal immune activation in late gestation increases neuroinflammation and aggravates experimental autoimmune encephalomyelitis in the offspring. Brain Behav Immun. 2015; 43: 159-71.

18. Kirsten TB, Lippi LL, Bevilacqua E, Bernardi MM. LPS exposure increases maternal corticosterone levels, causes placental injury and increases il-1 $\beta$ levels in adult rat offspring: relevance to autism. PLoS One. 2013; 8(12): e82244.

19. Krstic D, Madhusudan A, Doehner J, Vogel P, Notter $\mathrm{T}$, Imhof $\mathrm{C}$, et al. Systemic immune challenges trigger and drive Alzheimer-like neuropathology in mice. $\mathrm{J}$ Neuroinflammation. 2012; 9: 151. doi: 10.1186/17422094-9-151.

20. Vezzani A, Lang B, Aronica E. Immunity and inflammation in epilepsy. Cold Spring Harb Perspect Med. 2015; 6(2): a022699. doi: 10.1101/cshperspect. a022699.

21. Dambach H, Hinkerohe D, Prochnow N, Stienen MN, Moinfar Z, Haase CG, et al. Glia and epilepsy: experimental investigation of antiepileptic drugs in an astroglia/microglia co-culture model of inflammation. Epilepsia. 2014; 55(1): 184-92.

22. Janssen S, Schlegel C, Gudi V, Prajeeth CK, Skripuletz T, Trebst C, et al. Effect of FTY720- 
phosphate on the expression of inflammation-associated molecules in astrocytes in vitro. Mol Med Rep. 2015; 12(4): 6171-7.

23. Brambilla L, Guidotti G, Martorana F, Iyer AM, Aronica E, Valori CF, et al. Disruption of the astrocytic TNFR1-GDNF axis accelerates motor neuron degeneration and disease progression in amyotrophic lateral sclerosis. Hum Mol Genet. 2016; 15(14): 3080-95.

24. Kim D, Bae CH, Jun YL, Jeon H, Koo S, Kim S. Acupuncture alters pro-inflammatory cytokines in the plasma of maternally separated rat pups. Chin J Integr Med. 2017; 23(12): 943-7.

25. Yoo YM, Lee CJ, Lee U, Kim YJ. Neuroprotection of adenoviralvector-mediated GDNF expression against kainic-acid-induced excitotoxicity in the rat hippocampus. Exp Neurol. 2006; 200(2): 407-17.

26. Kanter-Schlifke I, Georgievska B, Kirik D, Kokaia M. Seizure suppression by GDNF gene therapy in animal models of epilepsy. Mol Ther. 2007; 15(6): 1106-13.

27. Sabaghi A, Heyrani A, Kiani A, Yousofvand N. Effect of aerobic training during pregnancy on seizureinduced depression in mouse. Shefaye Khatam. 2018; 6(3): $35-42$.

28. Kameda SR, Fukushiro DF, Trombin TF, ProcópioSouza R, Patti CL, Hollais AW, et al. Adolescent mice are more vulnerable than adults to single injection-induced behavioral sensitization to amphetamine. Pharmacol Biochem Behav. 2011; 98(2): 320-4.

29. Becker A, Grecksch G, Ruthrich HL, Pohle W, Marx B, Matthies H. Kindling and its consequences on learning in rats. Behav Neural Biol. 1992; 57(1): 37-43.

30. Saavedra A, Baltazar G, Duarte EP. Driving GDNF expression: the green and the red traffic lights. Prog Neurobiol. 2008; 86(3): 186-215.

31. Ibiza S, García-Cassani B, Ribeiro H, Carvalho T, Almeida L, Marques R, et al. Glial-cell-derived neuroregulators control type 3 innate lymphoid cells and gut defence. Nature. 2016; 21(535): 440-3.

32. Rickert U, Grampp S, Wilms H, Spreu J, KnerlichLukoschus F, Held-Feindt J, et al. Glial cell line-derived neurotrophic factor family members reduce microglial activation via inhibiting p38MAPKs-mediated inflammatory responses. J Neurodegener Dis. 2014; 369468 .

33. Nanobashvili A, Airaksinen MS, Kokaia M, Rossi j, Asztély F, Olofsdotter K, et al. Development and persis tence of kindling epilepsy are impaired in mice lacking glial cell line-derived neurotrophic factor family receptor a2. Proc Natl Acad Sci U S A. 2000; 97(22): 12312-7.

34. Barroso-Chinea P, Cruz-Muros I, Afonso-Oramas D, Castro-Hernández J, Salas-Hernández J, Chtarto A, et al. Long-term controlled GDNF over-expression reduces dopamine transporter activity without affecting tyrosine hydroxylase expression in the rat mesostriatal system. Neurobiol Dis. 2016; 88: 44-54.

35. Boger HA, Middaugh LD, Patrick KS, Ramamoorthy $\mathrm{S}$, Denehy ED, Zhu H, et al. Long-term consequences of methamphetamine exposure in young adults are exacerbated in glial cell linederived neurotrophic factor heterozygous mice. J Neurosci. 2007; 27(33): 8816-25.

36. Littrell OM, Pomerleau F, Huettl P, Surgener S, McGinty JF, Middaugh LD, et al. Enhanced dopamine transporter activity in middle-aged Gdnf heterozygous mice. Neurobiol Aging. 2012; 33(427): e1-14.

37. Giros B, Jaber M, Jones SR, Wightman RM, Caron MG. Hyperlocomotion and indifference to cocaine and amphetamine in mice lacking the dopamine transporter. Nature. 1996; 15(379): 606-12.

38. Salahpour A, Ramsey AJ, Medvedev IO, Kile B, Sotnikova TD, Holmstrand E, et al. Increased amphetamine-induced hyperactivity and reward in mice overexpressing the dopamine transporter. Proc Natl Acad Sci U S A. 2008; 105(11): 4405-10.

39. Boudanova E, Navaroli DM, Melikian HE. Amphetamine-induced decreases in dopamine transporter surface expression are protein kinase C-independent. Neuropharmacology. 2008; 54(3): 60512 .

40. Wayment HK, Schenk JO, Sorg BA. Characterization of extracellular dopamine clearance in the medial prefrontal cortex: role of monoamine uptake and monoamine oxidase inhibition. J Neurosci. 2001; 21: 35-44.

41. Lev N, Barhum Y, Pilosof NS, Ickowicz D, Cohen HY, Melamed E, et al. DJ-1 protects against dopamine toxicity: implications for Parkinson's disease and aging. J Gerontol A Biol Sci Med Sci. 2013; 68(3): 215-25.

42. Goldstein DS, Sullivan P, Cooney A, Jinsmaa Y, Sullivan R, Gross DJ, et al. Vesicular uptake blockade generates the toxic dopamine metabolite 3,4-dihydroxyphenylacetaldehyde in PC12 cells: relevance to the pathogenesis of Parkinson's disease. J Neurochem. 2012; 123(6): 932-43.

43. Masoud ST, Vecchio LM, Bergeron Y, Hossain MM 


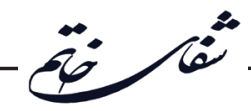

, Nguyen LT, Bermejo MK, et al. Increased expression of the dopamine transporter leads to loss of dopamine neurons, oxidative stress and L-DOPA reversible motor deficits. Neurobiol Dis. 2015; 74: 66-75.

44. Lohr KM, Bernstein AI, Stout KA, Dunn AR, Lazo $\mathrm{CR}$, Alter SP, et al. Increased vesicular monoamine transporter enhances dopamine release and opposes Parkinson disease-related neurodegeneration in vivo. Proc Natl Acad Sci U S A. 2014; 8(27): 9977-82.

45. Ho Y-H, Lin Y-T, Wu C-WJ, Chao Y-M, Chang AYW, Chan JYH. Peripheral inflammation increases seizure susceptibility via the induction of neuroinflammation and oxidative stress in the hippocampus. J Biomed Sci. 2015; 22(1): 46. doi: 10.1186/s12929-015-0157-8.

46. Labandeira-Garcia JL, Rodriguez-Pallares J, Dominguez-Meijide A, Valenzuela R, Villar-Cheda B, Rodriguez-Perez AI. Dopamine-angiotensin interactions in the basal ganglia and their relevance for Parkinson's disease. Mov Disord. 2013; 28(10): 1337-42.

47. Lee M. Neurotransmitters and microglial-mediated neuroinflammation. Curr Protein Pept Sci. 2013; 14(1): 21-32.

48. Underhill SM, Wheeler DS, Li M, Watts SD, Ingram SL, Amara SG. Amphetamine modulates glutamatergic neurotransmission through endocytosis of the excitatory amino acid transporter eaat 3 in dopamine neurons. Neuron. 2014; 83(2): 404-16.

49. Rahn KA, Slusher BS, Kaplin AI. Glutamate in CNS neurodegeneration and cognition and its regulation by GCPII inhibition. Curr Med Chem. 2012; 19(9): 1335-45.

50. Coulter DA, Eid T. Astrocytic regulation of glutamate homeos tasis in epilepsy. Glia. 2012; 60(8): 1215-26.
51. Hoerbelt P, Lindsley TA, Fleck MW. Dopamine directly modulates GABAA receptors. J Neurosci. 2015; 35(8): 3525-36.

52. Meldrum BS, Rogawski MA. Molecular targets for antiepileptic drug development. Neurotherapeatics. 2007; 4(1): 18-61.

53. Quilichini PP, Chiron C, Ben-Ari Y, Gozlan H. Stiripentol, a putative antiepileptic drug, enhances the duration of opening of GABA-A receptor channels. Epilepsia. 2006; 47(4): 704-16.

54. Rajabzadeh A, Bideskan AE, Fazel A, Sankian M, Rafatpanah H, Haghir H. The effect of PTZ-induced epileptic seizures on hippocampal expression of PSANCAM in offspring born to kindled rats. J Biomed Sci. 2012; 31: 56. doi: 10.1186/1423-0127-19-56.

55. Hammond MS, Sims C, Parameshwaran K, Suppiramaniam V, Schachner M, Dityatev A. Neural cell adhesion molecule-associated polysialic acid inhibits NR2B-containing N-Methyl-D- spartate receptors and prevents glutamate-induced cell death. J Biol Chem. 2006; 17(46): 34859-69.

56. Cossa AC, Lima DC, do Vale TG, de Alencar Rocha AK, da Graça Naffah-Mazzacoratti M, José da Silva Fernandes M, et al. Maternal seizures can affect the brain developing of offspring. Metab Brain Dis. 2016; 31(4): 891-900.

57. Saeedi Borujeni MJ, Hami J, Haghir H, Rastin M, Sazegar G. Evaluation of Bax and Bcl-2 proteins expression in the rat hippocampus due to childhood febrile seizure. Iran J Child Neurol. 2016; 10(1): 53-60.

58. Ghosh S, Jehi LE. New-onset epilepsy in the elderly: Challenges for the internist. Clev Clin J Med. 2014; 81(8): 490-8. 